\title{
Oral Submucous Fibrosis: Review on Mechanisms of Pathogenesis and Malignant Transformation
}

\author{
Rasika Priyadharshani Ekanayaka and Wanninayake Mudiyanselage Tilakaratne*
}

Department of Oral Pathology, Faculty of Dental Sciences, University of Peradeniya, Sri Lanka

\begin{abstract}
Studies focusing on epidemiology, histopathology and molecular biology/pathology on various aspects of oral submucous fibrosis (OSF), especially in the last decade have helped to understand the pathogenesis to a larger extent. In addition research in some aspects of carcinogenesis in the background of fibrosis has also advanced significantly in the recent past allowing us to understand the mechanisms involved in malignant transformation of the most prevalent oral potentially malignant disorder in South Asia. It has been shown that pathogenesis of OSF is directly related to arecoline present in arecanut and most of the alterations in various pathways and molecules leading to accumulation of collagen are mediated as a result of arecoline. Reduction of Matrix metalloproteinases (MMPs) and increased secretion of Tissue inhibitors of matrix metalloproteinases (TIMPs) play the most significant role in collagen accumulation whilst fibrogenic cytokines, mainly TGF- $\beta$ over expression leads to increased production of collagen. There are various other pathways/molecules contributing to the pathogenesis in varying capacities. Malignant transformation in OSF has also been studied by various groups in the recent past. Role of arecanut as a carcinogen is proven beyond doubt with a large number of animal studies demonstrating its carcinogenicity, mutagenicity and genotoxicity. Studies involved in many molecules implicated in cell cycle regulation, hypoxia, processes leading to DNA double strand breaks, senescence and many other pathways related to carcinogenesis have shown ample evidence for the arecanut induced malignant transformation in OSF. Some of the findings in these studies may be helpful in inventing new treatment strategies for a common disease without an effective treatment up to date. Further, the understanding of mechanisms of malignant transformation may lead to early diagnosis of oral squamous cell carcinoma (OSCC) arising in the background of OSF.
\end{abstract}

Keywords: Oral submucous fibrosis; TGF- $\beta$; Oral squamous cell carcinoma ; Mechanism of pathogenesis

\section{Introduction}

Oral submucous fibrosis, first described in the early1950s, is a potentially malignant disease predominantly seen in people of Asian descent. It is a chronic progressive disorder and its clinical features depend on the stage of the disease. The majority of patients present with an intolerance to spicy food, rigidity of lip, tongue and palate leading to varying degrees of limitation of opening of the mouth and tongue movement. The histopathological hallmark of the disease is fibrosis that affects most parts of the oral cavity, pharynx and upper third of the oesophagus. The disease is seen in countries where the habit of betel chewing is frequently practiced. The main aetiological agent causing the disease is confirmed as arecoline in arecanut. The prevalence of the disease is on the rise in South Asia in the recent years. The reason for the rapid increase of the disease is reported to be due to an upsurge in the popularity of commercially prepared areca nut preparations in South Asia. Although the disease was described in 1950 s, its pathogenesis and more importantly the mechanisms of malignant transformation have not been clear up to date. We produced a review paper on the pathogenesis of OSF in 2006 with the available research up to that time [1] However, a significant number of research carried out by our group and various other researchers since 2006 have helped to understand the pathogenesis and possible mechanisms of malignant transformation of the disease to some extent. Therefore, the aim of this review is to analyze critically the recent developments that may lead to our understanding of the pathogenesis (Figure 1) and malignant transformation of OSF (Figure 2). A special reference is also made to emphasize the fact that arecanut contains definite carcinogens, promoting oral carcinogenesis.

\section{Role of Arecoline as the Main Aetiological Factor}

The areca nut alkaloid arecoline is now identified as the principal causative factor for OSF. Effects of arecoline in the pathogenesis of OSF include fibroblastic proliferation and increased collagen formation. Many studies had been performed so far in order to find out the exact mechanism of arecoline in collagen metabolism.

Connective Tissue Growth Factor (CTGF) is associated with the onset and progression of fibrosis in many human tissues. In an attempt to analyze the expression of CTGF in OSF, it was found that CTGF was present in fibroblasts and endothelial cells in all the OSF cases included in the study. It was further shown that arecoline stimulated CTGF synthesis in a dose- and time-dependent manner in buccal mucosal fibroblasts through reactive oxygen species (ROS), NF-kappa $\mathrm{B}, \mathrm{JNK}$ and p38 MAPK pathway. In the mean time many studies have shown that NF-kappa B, JNK and p38 are strongly activated by ROS and thereby it is suggested that ROS elicited by arecoline may play important roles in NF-kappa B, JNK, P38 activations and subsequent CTGF expression. In conclusion the authors have hypothesized that overexpression of CTGF in arecanut chewing may enhance the fibrotic activity in OSF and thereby play a role in the pathogenesis of OSF. Unlike TGF $\beta$, CTGF is only produced by hepatic stellate and kidney mesangial cells in adults under normal conditions, therefore it may be used as a novel target for developing anti-fibrotic therapy in treating

${ }^{*}$ Corresponding author: WM Tilakaratne, Faculty of Dental Sciences, University of Peradeniya, Sri Lanka, Tel: 0094-812397435; Fax: 0094-812388948; E-mail: wmtilak@pdn.ac.lk

Received June 20, 2013; Accepted July 29, 2013; Published August 05, 2013

Citation: Ekanayaka RP, Tilakaratne WM (2013) Oral Submucous Fibrosis: Review on Mechanisms of Pathogenesis and Malignant Transformation. J Carcinogene Mutagene S5: 002. doi:10.4172/2157-2518.S5-002

Copyright: @ 2013 Ekanayaka RP, et al. This is an open-access article distributed under the terms of the Creative Commons Attribution License, which permits unrestricted use, distribution, and reproduction in any medium, provided the original author and source are credited. 


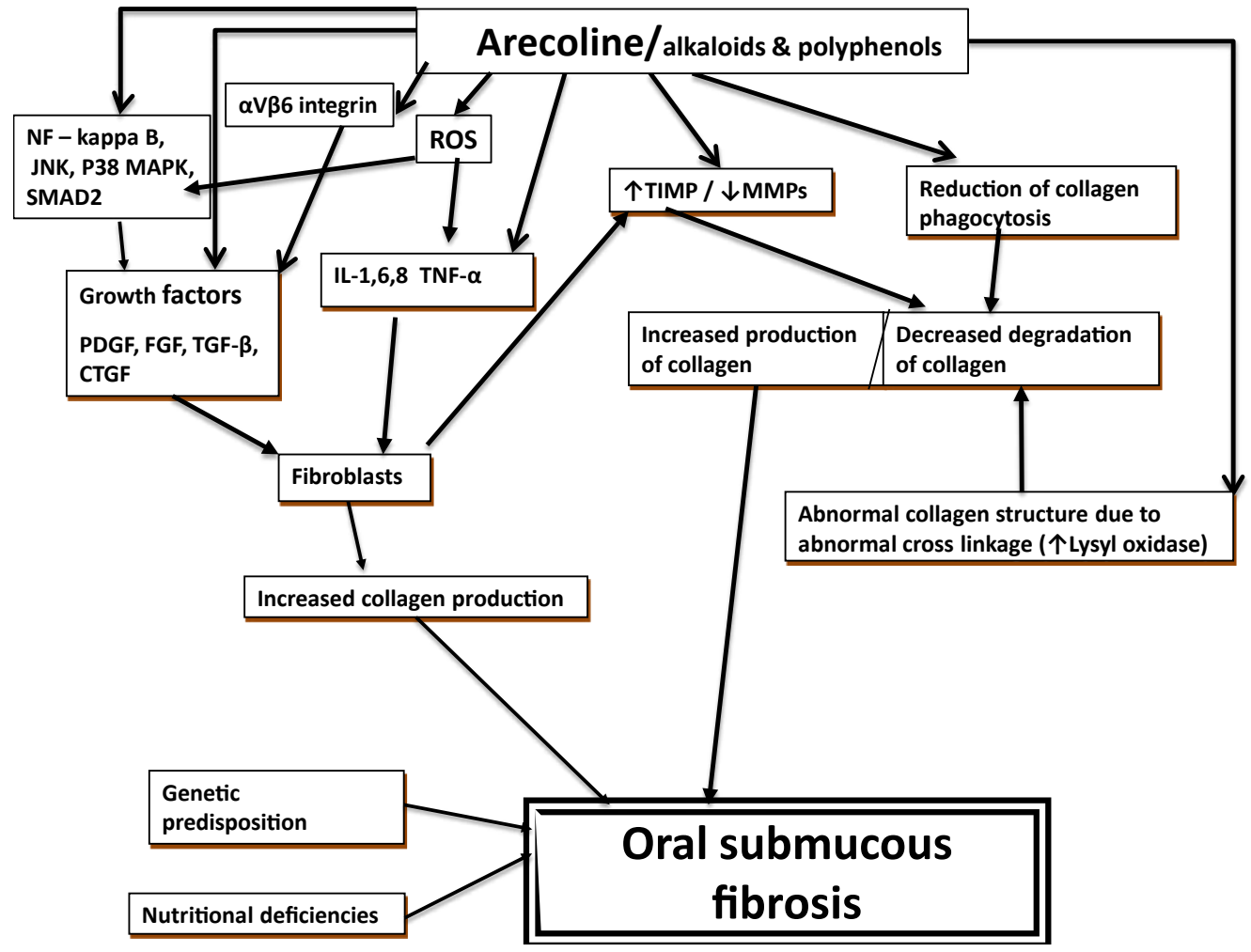

Figure 1: (Mechanisms of pathogenesis of oral submucous fibrosis) Possible molecules and pathways involved in the pathogenesis of Oral submucous fibrosis.

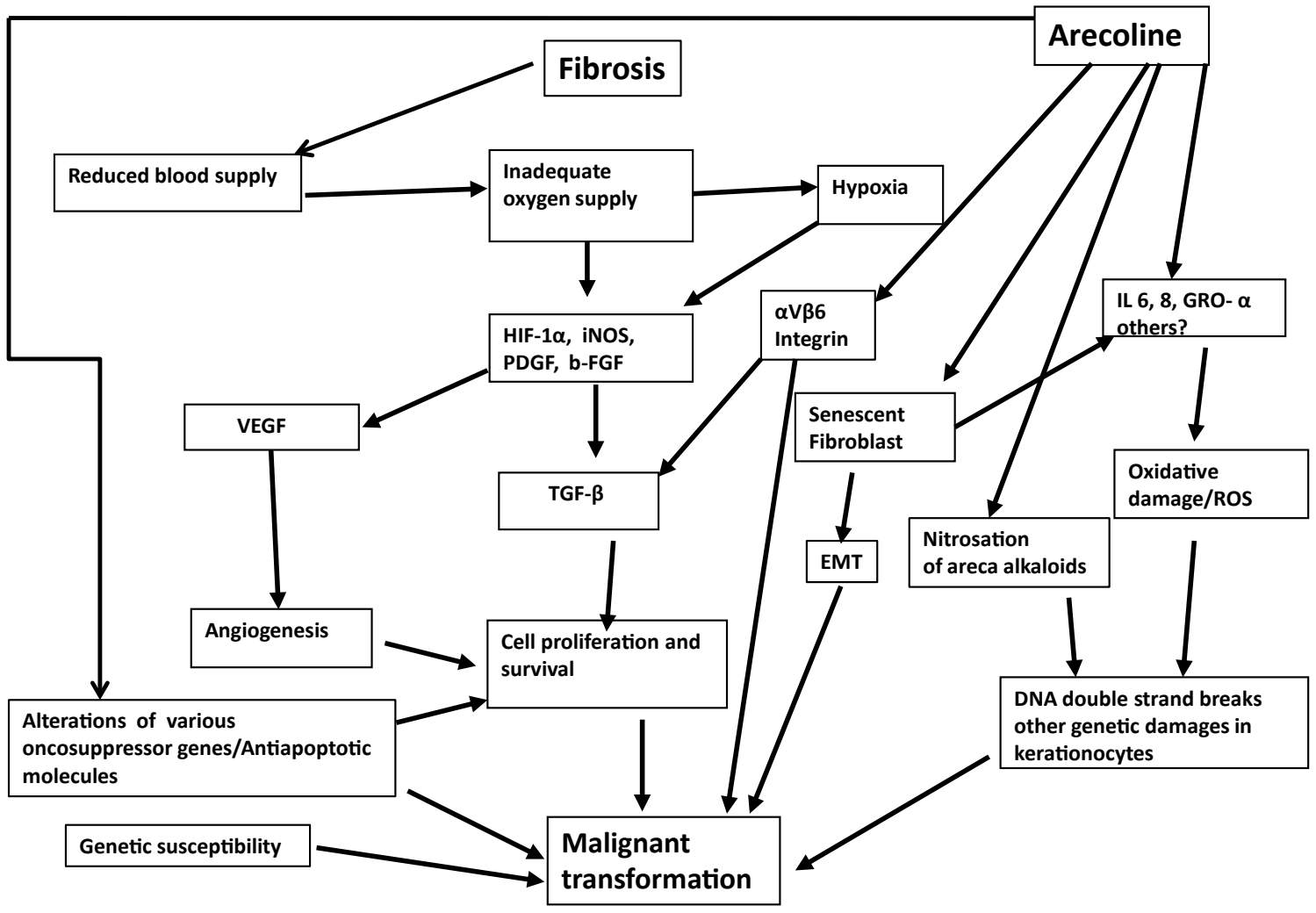

Figure 2: Possible events in malignant transformation of Oral submucous fibrosis. 
OSF. Inhibition of CTGF will block the profibrotic effects of TGF- $\beta$, without affecting its anti-proliferative and immunosuppressive effects [2].

Another study reports that arecoline influences deposition of extra cellular matrix (ECM) by increasing the production of TIMP-1. The effect is enhanced when fibroblasts are co cultured with keratinocytes, suggesting that interaction of oral keratinocytes and fibroblasts play an important role in the pathogenesis of OSF [3]. In addition, the interaction of arecoline and keratinocytes is reported to induce the differentiation of myofibroblasts from fibroblasts [4]. A different study suggests that areca alkaloids induce buccal mucosal fibroblast contraction and this process was related to phospholipase $\mathrm{C}$, inositol triphosphate $3, \mathrm{Ca}^{2+}$, calmodulin and Rho signaling pathway as well as actin filament polymerization. The persistent fibroblast contraction may induce fibrotic process in OSF. However, fibroblast contraction may not be purely dependent on ROS production [5]. The fact that arecoline influences ECM was further supported by the evidence for TGM-2 over expression in OSF and its regulation by arecoline. TGM2 stabilizes ECM protein by cross linking and has been implicated in several fibrotic disorders [6].

The impact of arecoline on endothelial cells has also been studied in detail. It is reported that at concentrations of 0.4 and $0.8 \mathrm{mM}$, arecoline induces cytotoxicity and also G2/M cell cycle arrest and increases sub- $\mathrm{G}_{0} / \mathrm{G}_{1}$ population, a hall mark of apoptosis. Further it is observed that prolonged exposure to arecoline $(0.1 \mathrm{mM})$ significantly suppresses endothelial cell proliferation. Also the exposure to $>0.2 \mathrm{mM}$ arecoline decreases the proportion of endothelial cells residing in $\mathrm{S}$ phase but increases the cells arresting in G2/M phase. These findings suggest that the anti-proliferative and cytotoxic effects of arecoline are possibly associated with the alteration of specific cell cycle regulatory proteins such as ATM, checkpoint kinases, p53, and cdc25C for G2/M checkpoint. Considering these findings the authors have suggested that the endothelial damage leading to impairment of vascular function contributes to the pathogenesis of OSF [7]. These findings explain the less vascularity that is observed in histological sections of OSF, especially with the progression of the disease. Further, that may explain the atrophy of the epithelium and hypoxic environment which may predispose them to carcinogenesis.

\section{Matrix Metalloproteinases and Tissue Inhibitors of Matrix Metalloproteinases (MMPs and TIMPs)}

OSF is histopathologically characterized by fibrosis of subepithelial connective tissue. Collagens are the major structural component of ECM, hence precise regulation of collagen metabolism is essential to maintain the normal integrity of connective tissue. OSF is not only due to excessive deposition but also due to decreased collagen degradation.

In OSF the equilibrium between MMPs and TIMP is disturbed in such a manner that it ultimately results in increased deposition of ECM. This concept is confirmed by several recent studies. One of our studies, with the use of immunohistochemistry identified MMP-1 expression to be attenuated in OSF compared to normal oral mucosa. Since MMP-1 is the main human enzyme that degrades fibrillar collagen, this suggests that collagen degradation caused by MMP- 1 is down regulated in OSF [8]. We also showed that there is no statistically significant difference in the fraction of TIMP-1 positive cells between OSF and normal oral mucosa, yet TIMP-1 shows stronger intensity in fibroblasts of OSF than in normal oral mucosa. Hence, collectively this study proved improper regulation of proteolytic equilibrium is one of the main factors responsible for the excessive fibrosis in OSF
[8]. The fibroblasts in OSF have a reduced replicative life span as they accumulate senescent cells during the progression of the disease. This is due to the increased amount of ROS and DNA double strand breaks (DDBs) produced intrinsically by damaged mitochondria. TIMP1 and 2 is increased in fibroblast cultures of OSF relative to normal and non diseased paan user controls. Following the introduction of unrepairable DDBs into normal oral fibroblasts TIMP-1 and TIMP2 secretion increased within 5 days, replicating early senescence and the elevation seen in OSF cultures. Thereby, it highlights the use of increased fibroblast TIMP-1 and 2 levels as effective markers of early OSF. Further studies regarding this finding have a clinical value as OSF may be reversed to normal at its early stage [9]. Another group has found that TIMP-1 production is enhanced when fibroblasts are co cultured with keratinocytes pretreated by arecoline. The authors have further suggested that interaction of oral keratinocytes and fibroblasts play an important role in pathogenesis of OSF [3]. It has been shown that endogenous collagenase activity in OSF tissues were 3-5 folds less than that in normal oral mucosa, which in turn might be one mechanism responsible for collagen accumulation [10]. A recent immunohistochemical study has reported a decrease in MMP-1 expression intensity in the epithelium and connective tissue of buccal mucosal tissue of OSF compared to normal. However no statistically significant difference was identified between the expression of MMP-1, among different histological grades of OSF [11].

All above studies provide evidence in support of the imbalance between MMPs and TIMPs via different mechanisms leading to accumulation of collagen which is the hallmark of the disease. Further, the interaction between keratinocytes and fibroblasts is important not only in pathogenesis but also in malignant transformation as we have shown that OSF is an epithelial driven connective tissue disease, with some of our studies discussed below. ROS related DNA DDBs appeared to play a role in both pathogenesis and malignant transformation.

Establishment of the balance between MMPs and TIMPs should be the target to achieve in the management of OSF. Although, various studies have tried to target MMP inhibitors for cancer treatment $[12,13]$, in the context of OSF, we could not find studies trying to target molecules such as MMP and TIMP to either increase collagen breakdown by increasing MMP levels or try to increase the level of MMPs by blocking TIMPs. Further, to date no promising TIMP inhibitor is identified and further research should be carried out in this regard.

\section{Copper and Related Structural Changes of Collagen}

Arecanut is high in Copper, and its role in pathogenesis of OSF has been subjected to a wide array of studies [14]. The enzyme lysyl oxidase is a copper activated enzyme critical for collagen cross linking and organization of ECM [15]. Salivary Copper is found to increase markedly, following chewing arecanut. This finding indicates that soluble Copper found in arecanut is released into the oral environment and its buccal absorption may contribute to fibrosis of buccal mucosa. This suggests the local effect of Copper in OSF patients [16]. Furthermore the use of saliva as an effective tool in screening of both OSF and OSCC has been highlighted. There is an increase in salivary Copper levels from mild OSF to severe cases which are graded histopathologically [17]. Another group has demonstrated increased serum Copper levels in OSF suggesting a systemic effect. It has further reported an increase in serum Copper level as the clinical stage of OSF increases $[18,19]$ Taking all the studies on copper in to account, the effects appear to be local in the context of OSF as there is no evidence 
to suggest that these patients showing any clinical or experimental evidence of systemic fibrosis.

\section{Accumulation of Collagen and Other Changes in Extracellular Matrix}

There had been several attempts to identify the dynamics of ECM remodeling with the progression of the disease. One of our investigations revealed that in the early stage of OSF tenascin, perlecan, fibronectin and collagen type III were enhanced in the lamina propria and submucosal layer. In the intermediate stage elastin was extensively and irregularly deposited around muscle fibres, together with the above mentioned molecules. In the advanced stage all those ECM molecules decreased and were entirely replaced by collagen type I only. Their gene expression levels were varied with the progression of fibrosis. Therefore, it is clear that ECM remodeling steps in OSF are similar to each phase of usual granulation tissue formation and maturation. In addition, clinical presentation of trismus may be related to loss of variety of ECM molecules including elastin and replacement of muscle fibres by homogenous collagen type I [20].

Since OSF is considered as a disease of altered collagen metabolism, collagen and associated factors had been studied in detail during the recent years. Heat shock protein (HSP 47) is a collagen specific molecular chaperone involved in the processing and/or secretion of procollagen. HSP 47 is significantly upregulated in OSF. It is a known collagen specific molecular chaperone involved in the processing and/ or secretion of procollagen hence its role in OSF has been subjected to experiments by treating fibroblast with arecoline Arecoline was found to elevate HSP $47 \mathrm{~m}$-RNA expression in fibroblasts, in a dose dependent manner through MEK, PI3K and COX-2 signal transduction pathways [21]. Cystatin C, a non glycosylated basic protein is increased in a variety of fibrotic diseases. In a similar manner as HSP 47, Cystatin $\mathrm{C}$ was found to be upregulated both at $\mathrm{m}$-RNA and protein levels in the disease. Arecoline is responsible for this enhancement in a dose dependent manner [22]. Malondialdehyde (MDA) is a lipid peroxidation end product with the potential to stimulate fibroblasts and to increase collagen production by 2-3 times. A recent study has reported a significant elevation in serum MDA levels as the grading of OSF progressed. Tissue MDA levels were increased in grade 1 and 2 OSF when compared to controls. Yet tissue levels of MDA were found to be reduced in grade 3 OSF when compared to controls, suggesting the utilization of MDA in crosslinking of collagen at the advance stage of the disease [23].

\section{Growth Factors and Inflammatory Cytokines}

Considerable advances have been made in the understanding of growth factors and inflammatory cytokines associated with OSF. In an attempt to understand the changes in cytokine levels, gene expression profiling was performed in OSF tissues. The results revealed upregulation of an array of cytokines namely, TGF $\beta$ 1, TGF $\beta$ 1p, THBS1, SPP1, TIG1, TGM2 and CTGF and down regulation of BMP7 which is a known negative modulator of fibrosis [24]. TGF $\beta$ is considered to be a potent stimulator of production and deposition of ECM [25]. It is the main trigger for the increased collagen production and decreased matrix degradation pathways in OSF [26]. It has been shown that the expression of TGF- $\beta 1$ was significantly upregulated in the connective tissue portion of the disease compared to normal [8]. The role of activation of TGF $\beta$ signaling as the main causative event for increased collagen production in OSF was further illustrated by a study of global gene expression profile in oral epithelial cells. Treatment of cells with arecanut water extract consisting of polyphenols and alkaloids, has shown that $64 \%$ of the differentially regulated genes by arecanut water extract matches with the TGF $\beta$ induced gene expression profile [27]. This suggests that arecoline induces TGF $\beta$ in epithelial cells and furthermore this is observed only in epithelial cells but not in human gingival fibroblast cells, indicating that epithelial cells could be the source of TGF $\beta$ in OSF. We have confirmed the above finding with one of our studies, that OSF is a epithelial driven disease as the early signals are given to epithelial cells to secrete TGF $\beta$ through $a v \beta 6$ integrin and the expression of TGF $\beta$ disappears when the drug tropicamide blocks $\alpha v \beta 6$ integrin [28]. Phosphorylation of SMAD-2 was observed following treatment of epithelial cells by catechin, tannin and alkaloids, hence the authors state that arecanut mediated activation of p-SMAD-2 involves upregulation and activation of TGF $\beta$ [27]. $\mathrm{CTGF} / \mathrm{CCN} 2$ and COX-2 were found to be over expressed in OSF. The CCN2 synthesis in buccal mucosal fibroblasts is stimulated by TGF $\beta 1$ and this reaction is mediated via ALK5, JNK and p38 MAPK pathways. Epigallocatechin-3-gallate (EGCG) in turn can completely block TGF $\beta 1$ induced CCN2 synthesis by suppressing JNK and p38 in buccal mucosal fibroblasts, which may be useful in controlling OSF [26]. Also the TGF $\beta 1$ induced CCN2 synthesis in buccal mucosal fibroblasts is not inhibited by PGE2, as in fetal lung fibroblasts. Hence the CTGF/ CCN2 levels are maintained in the OSF tissue in the presence of COX2 [26]. In an attempt to correlate TGF $\beta$ with loss of adipose tissue in OSF, it was found that TGF $\beta$ plays a key role in causing lipodystrophy. In addition it reports that TGF $\beta$ secretion is more during the early course of the disease than in the advanced stage [29]. b-FGF is another cytokine found to be increased in the progression of the disease. It is increased in fibroblasts and in endothelial cells in early disease, and in advanced fibrosis b-FGF expression was noted more in stroma. Authors have related the increase expression in early stages to an initial injury phase due to areca consumption [30]. Another group of researchers has reported that IGF-1 expression is significantly upregulated in OSF both at m-RNA and protein levels. Arecoline had been responsible for this elevation in a dose dependant manner. It is found to be expressed mainly by fibroblast, endothelial cells and inflammatory cells [31]. Among the many cytokines implied in the pathogenesis by influencing the synthesis of collagen, Tumor Necrosis Factor- $\alpha$ (TNF- $\alpha$ ) is of critical importance. TNF- $\alpha$ mediates multiple functions, out of which the regulation of inflammatory reaction as well as transcription of collagen and collagenase are the most important in the pathogenesis of OSF. Recently it was reported that the homozygous wild genotype TNF- $\alpha 2$ was significantly associated with an increased risk of OSF and the mutant allele TNF- $\alpha 2$ is about 7 times more efficient in promoter function than the wild allele. Considering these facts it was suggested a role of TNF- $\alpha$ in OSF pathogenesis through its modulation of collagen metabolism [32]. With the available literature on growth factors and cytokines, TGF $\beta$ appears to be the main mediator of the disease and others such as TNF- $\alpha$, IGF-1, b-FGF and CTGF may contribute to continuous accumulation of collagen with activation of signaling pathways such as ALK5, JNK, SMAD and p38 MAPK. These molecules and pathways can be the target areas for new treatment strategies.

\section{av $\beta 6$ Integrin and Pathogenesis}

av $\beta 6$ integrin was found to be markedly upregulated in OSF. In vitro studies have demonstrated that $\operatorname{av} \beta \quad 6$ activates TGF- $\beta 1$, the principal modulator of tissue fibrosis. TGF- $\beta 1$ in turn induces transdifferentiation of oral fibroblasts into myofibroblasts, and upregulates the genes associated with tissue fibrosis. Myofibroblasts facilitate the matrix accumulation both by increasing the deposition of matrix proteins mainly in the form of collagen I and by decreasing 
the degradation of matrix proteins by up-regulating the matrix metalloproteinase inhibitor TIMP-1. The above in vitro findings were confirmed using human clinical samples by one of our studies. It was demonstrated that the fibrosing lamina propria contained SMApositive myofibroblasts and TGF- $\beta 1$ dependent SMAD signaling was detectable in those myofibroblasts confirming the activation of TGF- $\beta$ 1. Nuclear positivity for phosphorylated SMAD 2 was found in lesional keratinocytes as well, suggesting the role of TGF- $\beta 1$ in inhibiting the epithelial growth and thereby causing pronounced epithelial atrophy in OSF. Analyzing the findings of the entire study we concluded that the pathogenesis of OSF may be epithelial-driven and involve arecoline-dependent up-regulation of $\operatorname{av} \beta \quad 6$ integrin [28]. av $\beta 6$ integrin is discussed in more detail later in the review under malignant transformation.

\section{Epithelial-Mesenchymal Transition (EMT)}

Epithelial-Mesenchymal Transition (EMT) has been a clearly recognized process for decades in developmental biology and it has gained much attention recently due to its important implication in cancer and fibrosis. In a study designed to analyze the role of EMT in the pathogenesis of OSF three major events involved in EMT of OSF were identified. Cell injury caused by Areca Nut Extract (ANE) produces ROS which in turn triggers both MAPK and NF- $\kappa B$ pathways involved in EMT of OSF. Thereby it has been demonstrated that exposure to ANE causes alterations of normal keratinocyte morphology and it is also able to induce the cell cycle arrest at G1/S phase and the senescence-associated phenotypes. Furthermore keratinocytes secrete a variety of inflammatory mediators such as PGE2, IL-6, TNF- $\alpha$ and most importantly TGF- $\beta$, in response to injury [33]. Hif- $1 \alpha$ enhances the EMT in vitro and promotes fibrogenesis by increasing expression of extracellular matrix-modifying factors and lysyl oxidase genes [34]. A study which was carried out using alveolar epithelial cells has shown that both ROS and HIF- $\alpha$ were necessary for hypoxia-induced TGF- $\beta 1$ upregulation [35]. It is reasonable to hypothesize a possible relationship between Hif- $1 \alpha$, ROS and EMT in OSF as we have convincingly shown that upregulation of Hif- $1 \alpha$ in the disease and production of ROS by arecoline treatment [36].

All these events collectively contribute to EMT in OSF and lead to fibrosis. Potentially, the transformed cell may proliferate to expand the fibroblast population, undergo apoptosis, or revert back to epithelial type. The fact that the cell is able to dedifferentiate back to epithelial form directs future research into a novel path as it hints towards a possibility of reversing the disease process [31].

\section{Genetic Polymorphism Predisposing to OSF}

A wide spectrum of chromosomal, genetic and molecular alterations is associated with both pathogenesis and malignant transformation of OSF, yet majority remains unclear [32]. In an attempt to identify the genes responsible for pathogenesis and malignant transformation of OSF using oligonucleotide microarray, 716 genes were found to be upregulated and 149 genes were downregulated in OSF. Gene expression profiles of normal and OSF were clearly different. It is identified that genes are involved in immune response, inflammatory response and EMT induced by TGF $\beta$ signaling pathway namely SFRP4. THBS1, MMP2, ZO-1, and CK18 were differentially expressed in the disease suggesting its importance in pathogenesis and also malignant transformation [33]. In another study, differentially expressed genes in OSF had been analyzed using bio informatic tools. A majority of the differentially expressed genes were located on chromosome 1,2,5,6,7,11 and 12. Gene Ontology (GO) classification of these genes identified cellular component sub groups associated with extra cellular matrix, cytoskeleton and cell membrane and also biological process subgroups associated with protein binding, signal transducer activity, immune and defense responses [34]. Buccal mucosal cells in patients with the disease exhibited cytological alterations especially micronuclei and other nuclear anomalies as binucleation, karyorrhexis and karyolysis, reflecting the genotoxic and cytotoxic properties associated with betel quid chewing [35]. Similar findings were reported in a study based on human lymphocytes further emphasizing the results of the earlier study [36]. Polymorphisms of various genes have contributed to the pathogenesis in different aspects. Cytochrome P450 gene family plays a major role in oxidative metabolism of active endogenous and xenobiotic substrates. Genetic predisposition in pharmacokinetics for toxic substances of betel quid plays a major role in development of the disease. Meanwhile polymorphism of Cytochrome P450 3A gene family is considered as a major determinant of the interindividual variability in chemical pharmacokinetics. Hence Cytochrome P450 had been identified as a genetic biomarker for susceptibility to OSF and authors have further suggested that individuals at genetically high risk for OSF could be screened according to the genetic polymorphisms in some exclusive regions of the Cytochrome P450 3A genes [37]. Another study highlighted that polymorphism in Cytochrome P4501A1 and CYP2E1 may confer an increased risk for OSF [38]. While a different study brought about the fact that CYP1A1 (m1) genotype and (m2) genotype singly acts as a protective factor but in the absence of GSTM1 and/or GSTT1 gene significantly alters risk towards the disease [39]. Genes [CYP2B6, CYP2C18, CYP2F1, CYP3A5, microsomal glutathione S-transferase 2 (MGST2), alcohol dehydrogenase (ADH), UDP glucuronosyl transferase 2B15 (UGT2B15), ADH1C) which were related to the pathway of CYP metabolism were found to be down regulated in all stages of OSF, thereby reducing the ability of CYP to metabolize and clear betel nut substances. This may ultimately contribute to the pathogenesis [40]. Recently, genetic polymorphism of lysyl oxidase, an enzyme essential for collagen cross linking was identified and LOX Arg158GIn was encountered more in elderly OSF patients [13]. Effects of polymorphisms of collagen-related genes on OSF risk had been studied in detail. A study focused on the Single Nucleotide Polymorphisms (SNPs) of TGF $\beta-1$ gene reported that polymorphism in 5 \&UTR C-T in TGF- $\beta 1$ gene has a significant association with OSF, as it is a main determinant in the pro-angiogenic pathway which is important in the pathophysiology of the disease [41]. A research group analyzing the association of SNP $(-11715 \mathrm{~A}->6 \mathrm{~A})$ in the MMP-3 promoter region of OSF, had finally concluded that the expression of MMP-3 genotype associated with the 5A alleles has an increased risk for developing the disease [42]. However, another study has illustrated that the SNPs in the MMP-2 and MMP-9 promoter region is not associated with susceptibility to OSF [43]. In an attempt to assess the association between OSF and TNF- $\alpha$ genetic polymorphism $(-308)$, it was found that the genotype distribution of TNF- $\alpha(-308)$ genetic polymorphism show similar distribution among areca chewers and non-areca-chewers implicating that the habit of areca-nut chewing is not associated with TNF- $\alpha(-308)$ genetic polymorphism [30]. N-acetyltransferase 2 locus codes for an enzyme that catalyzes acetylation of aromatic amines. Polymorphisms at this specific site can cause improper acetylation of the amines, leading to DNA adduct formation. It is suggested that these polymorphisms can increase the risk of OSF in men if exposed to arecanut or smokeless tobacco usage [44]. These studies provide significant evidence to support the theory of possible genetic predisposition to the disease. However, in order to confirm these findings, studies using larger samples of patients should be carried out. 
The impact on cell cycle regulation in OSF had been investigated. One such study demonstrated that ROS generated by arecoline may cause cell cycle arrest at the G1/G0 phase in human keratinocyte cells without affecting the expression of p21/Cip1. Oxidative stress may induce epithelial cell death without eliciting apoptosis at higher arecoline concentration. Meanwhile sub-lethal concentrations of arecoline upregulated the expression of several stress responsive genes namely; heme oxygenase-1, ferritin light chain, glucose-6phosphate dehydrogenase, glutamate-cysteine ligase catalytic subunit and glutathione reductase [45]. However, a similar investigation reported that $0.01-0.04 \mathrm{mM}$ arecoline caused late-S and $\mathrm{G} 2 / \mathrm{M}$ phase cell cycle arrest in human KB epithelial cells. A decrease in cdc2 and Cyclin B1 protein levels and increasing p21 were identified in gingival keratinocytes by Western blot analysis. The same study revealed that inhibition of $\mathrm{KB}$ epithelial cell growth in a dose and time dependant manner and a reduction in cell number with higher arecoline concentration. Further increase in arecoline concentration has induced both cell necrosis and apoptosis [46].

\section{Malignant Transformation of Oral Submucous Fibrosis}

OSF is a well recognized potentially malignant disorder of the oral mucosa. Paymaster first described the malignant potential of OSF in 1956, the rate of which has been estimated to be $7-13 \%$ recently. Many follow up studies had been conducted so far in order to identify the important aspects in malignant transformation of OSF [47]. Recently it has been proposed that oral cancers arising in OSF constitute a clinicpathologically distinct disease, the differences of which believed to arise from differential mechanisms of arecanut carcinogenesis. One study recognized that most of these patients are younger males with better prognostic factors such as better grade of tumour differentiation, lesser incidence of nodal metastases, and extracapsular spread [48]. Another retrospective study in China has reported contradictory data. They state that OSCC originated from OSF is clinically more invasive and also exhibits higher metastasis and recurrence rate than OSCC not originated from OSF [49]. Our experience in reporting large numbers of OSF and also OSCC arising from the disease is that clinical presentation and behavior have a wide spectrum. Minority of cases during the process of transformation show large exophytic lesions which are clinically typical OSCC without showing any histological evidence of invasion.

This raises the question, whether the ECM in at least a subset of OSF is different and resistant to normal invasive mechanisms. This area needs further investigation.

Simultaneous occurrence of Oral Leukoplakia (OLE) and OSF is demonstrated to carry a higher risk for OSCC than those with either OLE or OSF alone, and they are reported to experience malignant transformation early [50]. Wide array of studies had been implemented recently in order to determine the possible mechanisms involved in malignant transformation and also many have focused their attention on molecular markers which could be helpful in early diagnosis and expecting therapeutic implications for carcinogenesis in the background of OSF (Figure 2).

\section{Arecanut as a Carcinogen}

Carcinogenicity of arecanut without tobacco is a well recognized fact by now [51] and arecoline the major alkaloid of arecanut is reported to have various cytotoxic and genotoxic properties. A recent study has proposed arecoline $\mathrm{N}$-oxide, the active metabolite of arecoline, as the ultimate carcinogen in areca related oral carcinogenesis. This conclusion was based on the fact that arecoline $\mathrm{N}$-oxide was able to cause mutagenicity in Salmonella typhimurium tester strains used [52]. Areca nuts and its constituents exhibit various modes of actions in inducing malignant transformation of OSF and many studies had been carried out in order to recognize them precisely. One such study reports that arecoline being a potent carcinogen, it downregulates p21 and p27 through reactive oxygen species/mTOR complex 1 (ROS/ mTORC1) pathway. The reduced levels of p21 and p27 might facilitate G1/S transition of the cell cycle and subsequently may lead to errorprone DNA replication [53]. A different study using Western blot analysis reported a decrease in cdc2 and Cyclin B1 protein levels and an increase in p 21 in gingival keratinocytes and vice versa in $\mathrm{KB}$ cancer cells following exposure to arecoline. This further emphasized the fact that regulation of $\mathrm{S}$ and/or $\mathrm{G} 2 / \mathrm{M}$ cell cycle related proteins play a vital role in different stages of arecoline mediated carcinogenesis [46]. The ability of arecoline to induce alterations in various genes had been studied in detail. The expression of Brcal and Brca2 tumour suppressor genes is reduced [54] in OSF while human telomerase reverse transcriptase (hTERT) is overexpressed [55]. Therefore, both events may contribute to malignant transformation of OSF. A study from our group showed that arecoline upregulates $\alpha v \beta 6$ expression in oral keratinocytes which in turn promotes keratinocyte migration and induce invasion [26]. As a response to arecoline, COX-2 gene is up regulated in fibroblasts, mainly at the early stage of the disease. COX-2 receptors are present on neoplastic cells and COX-2 gene expression is more pronounced in OSCC when compared to premalignant lesions. This may suggest a role of COX-2 receptors in carcinogenesis [56]. Arecanut has a high Copper content which activates several angiogenic factors such as VEGF, TNF $\alpha$, IL-1 and b- FGF. These molecules stimulate endothelial cell proliferation and activation. Hence Copper is recognized to play an important role in tumour angiogenesis. Both serum and salivary Copper levels are reported to be high in patients with oral cancer. Furthermore, salivary Copper levels are higher in oral cancer than in patients with OSF. These findings are suggestive of a possible influence of Copper in malignant transformation of OSF [15]. Presence of direct acting alkylating agents in arecanut, at levels that are sufficient to cause DNA damage has also been reported. Although these direct-acting methylating and ethylating agents have not been identified yet, this study directs our attention towards another possible mechanism of areca nut-mediated oral carcinogenesis [57]. It has been shown that ROS produced during auto oxidation of arecanut polyphenols in betel quid chewer's saliva, play an important role in the initiation and promotion of oral cancer. Arecanut specific nitrosamines produced by nitrosation of areca alkaloids are found to induce tumors in experimental animals and furthermore arecaidine and arecanut extracts are demonstrated to be tumor promoters. However, antioxidants as glutathione and $\mathrm{N}$-acetyl-L-cystine have the potential to prevent the damage of cellular macromolecules (DNA, proteins, and membrane lipids) by cytotoxic agents described above. Yet, repeated and continuous exposure of oral mucosal cells to an excessive amount of ROS, methylating agents and reactive metabolic intermediates from betel quid can damage cellular DNA [58]. However, it has been suggested that arecanut toxicity is not completely due to its polyphenols, tannin and alkaloid content, therefore further experiments are necessary to clarify agents responsible for arecanut carcinogenicity [58].

One of our most recent studies investigated the role of cytokines in arecanut related carcinogenesis, targeting the malignant transformation of OSF. For this study, normal human gingival fibroblasts (hNOF) and immortalized hNOF (hTERT-hNOF) were used. Cytokine antibody array was employed to screen cytokines secreted from fibroblasts 
exposed by arecanut (AN). We found that the levels of GRO- $\alpha$, IL-6, and IL-8 were increased in AN treated fibroblasts and these results were confirmed by ELISA and immunocytochemistry. Immortalized human oral keratinocytes (IHOK) were treated with these cytokines or conditioned media (CM) and assessed for ROS generation, oxidative DNA damage, DNA DDB and nuclear ploidy change. These approaches showed that ROS-generating cells were 7 or 8 -fold higher in cytokine-treated IHOK compared to non-treated IHOK. The positive cells showing DNA DDB and 8-oxoG FITC conjugate increased after treatment with cytokines or $\mathrm{CM}$ and could be reduced by neutralizing cytokines or anti-oxidant treatment. Furthermore, the nuclear aneuploid cells were 2-fold increased in cytokine-treated IHOK. OSF tissues also showed cytokine expression, oxidative DNA damage and DNA DDB. Taken together, we showed that the fibroblasts exposed to AN caused DNA damage in IHOK through cytokine release, suggesting a contributory mechanism to malignant transformation in OSF. This study provides insights to develop novel preventive modalities by inhibiting their routes of transformation [59].

\section{Hypoxia}

A detail study from our group based on the hypothesis that hypoxia plays a role in malignant transformation and progression of OSF, has identified that HIF-1 $\alpha$ is upregulated at both protein and mRNA levels. This has also been shown in cancers from different sites such as breast and urinary bladder. We found a statistically significant correlation with the degree of epithelial dysplasia and expression level of the gene HIF-1a. Along with the fact that HIF-1 $1 \alpha$ is a known transcription factor induced by hypoxia, we proposed that hypoxia together with HIF$1 \alpha$ play a role in malignant transformation of OSF. The possibilities of using HIF-1a as a marker for malignant transformation have also been discussed [60]. Another study from our group designed to evaluate the relationship between thickness of fibrosis and epithelial dysplasia in OSF provides more evidence to support our hypothesis of hypoxia and the malignant transformation. After analyzing 107 biopsies with histopathologically confirmed OSF we concluded that the advancement of fibrosis increases the risk of development of epithelial dysplasia. Increased fibrosis of the connective tissue causes reduction of vascularity resulting in hypoxia and subsequent overexpression of HIF-1 $\alpha$. This study provides the insight that the reduction of excessive fibrosis by appropriate treatment might help to reduce the occurrence of epithelial dysplasia, and ultimately, the development of OSCC [61].

\section{Alterations in Cell Cycle}

A recent study has revealed that proliferating activity as denoted by Proliferating Cell Nuclear Antigen (PCNA) index was higher in OSF epithelium than normal oral mucosa. Furthermore the PCNA index of the dysplastic OSF was significantly increased when compared with the non-dysplastic group. Analyzing all their findings, it was suggested that the increased PCNA index may correlate with the increased malignant transformation potential [8]. In a study designed to evaluate the important molecules in G2/M phase namely Cyclin B1, p34 (cdc2) and p-survivin, it was revealed that their expression is higher in OSF than in normal. Further significant difference was present in expression between OSF and OSCC. Authors have suggested that above mentioned molecules may play a key role in carcinogenesis by influencing mitosis [62].

Survivin is a protein expressed in a cell cycle dependent manner, mainly in G2/M phase and it is involved in inhibition of apoptosis while promoting cell division. Survivin Thr34 phosphorylation is the crucial requirement for survivin function. In an attempt to determine the potential involvement of survivin Thr34 phosphorylation in carcinogenesis of OSF, results indicate that the survivin phosphorylation on Thr34 in OSCC originated from OSF group was significantly higher than that in OSF group and on the same time absent in normal oral mucosa. These findings are consistent with the results of the previous study described above. It suggests that during carcinogenesis, phosphorylation of survivin on Thr34 increase gradually yet there is no relationship between the degree of surviving Thr34 phosphorylation and the stages of OSF. A gradual increase in expression of Cyclin B1, p34cdc2 and p-34cdc2 was observed in carcinogenesis of OSF suggesting an acceleration of cell proliferation during the process. Furthermore, p34cdc2-cyclin B1 kinase was confirmed to phosphorylate survivin on Thr34 in malignant transformation. It was concluded that phosphorylation of survivin on Thr34 critically regulates survivin and plays an important role during the malignant transformation of OSF both by inhibition of apoptosis and encouraging mitosis in carcinogenesis [63]. Later, there had been attempts to analyze the relationship between the survivin expression and clinical characteristics of the disease. It was shown that survivin expression levels were significantly higher in the OSCC transformed from OSF compared with OSF and normal. Though survivin expression exhibited differences in different stages of the disease, no relationship was found between the degree of survivin expression and the stages of OSF. Furthermore there was no relationship between survivin expression and the stage of OSCC transformed from OSF [64]. These studies confirm the value of survivin as both prognostic and predictive marker in malignant transformation of OSF. Therefore, we strongly suggest planning more studies using larger samples to investigate the role of survivin as a marker for early diagnosis of carcinogenesis in OSF.

\section{Alterations in Oncosuppressor Genes and Other Genes}

It was evident in earlier sections the role of some oncosuppressor genes in areca related carcinogenesis [46,53]. In the evaluation of PTEN, a known tumour suppressor gene, a progressive reduction of PTEN expression was noticed as normal mucosa changes into OSF and then into OSCC. Hence alteration of PTEN is considered as an important molecular event in pathogenesis and carcinogenesis [65]. Brcal and $\mathrm{Brca} 2$ are tumour suppressor genes involved in the maintenance of genomic integrity by facilitating error free DNA repair. A study which exposed mice to arecanut extract chronically and transgenerationally reports a decline in Brca1 and Brca2 proteins thus causing a genomic instability. Furthermore mutation in exon 11 of Brcal gene carried increased risk of cancer [54]. Arecoline increases the expression of hTERT in both mRNA and protein levels in oral keratinocytes. This over expression is supposed to have an important role in malignant transformation of OSF [55]. FHIT (Fragile Histidine Triad) is expressed in the epithelium of normal oral mucosa and a decrease in the expression of FHIT was noticed in OSF and more significantly in carcinoma arising in OSF. In contrast, MDM2 expression was negative in normal oral mucosal epithelium and an increased expression was seen in OSF and more significantly in carcinoma arising in OSF. Loss of FHIT and overexpression of MDM2 are stated to play an important role in carcinogenesis of the disease [66]. Over expression of Loricin and loss of Cytochrome P450 3A5 (CYP 3A5) are involved in the change of defending ability of epithelium, hence it is suggested that they may be responsible for carcinogenesis of OSF [67]. Hypermethylation of E-cadherin and COX-2 is a mutation of epigenetic levels and was observed in $52 \%$ and $30 \%$ of the patients' respectively. Chronic OSF was significantly associated with hypermethylation which may suggest 
that they are more prone to malignant transformation [68]. In contrast to the fact that COX-2 receptors play a role in carcinogenesis, COX-2$765 \mathrm{G}>\mathrm{C}$ polymorphism was identified as a protective factor against OSCC development [69]. However, there was no association between OSCC arising in OSF patients and TNF a (-308) genetic polymorphism [30].

\section{Angiogenesis Related Molecules}

It is conventionally accepted that atrophic epithelium in OSF is a result of decreased vascularity in the underlying connective tissue stroma. The consequent lack of tissue perfusion is believed to trigger the ischemic atrophy of epithelium and thereby making it vulnerable to the effects of oral carcinogens. There are many studies with diverse findings published regarding the degree of vascularity of OSF mucosa.

One such group has designed a study for characterization and quantification of mucosal vasculature and its possible role in malignant transformation of atrophic epithelium with the use of immunohistochemical marker, CD34. They forward scientific data to prove OSF epithelium as atrophic since histopathologically all the cases showed reduced epithelial thickness when compared to controls. They quantified vasculature in the form of MVD (mean vascular density) and considered it as the index of angiogenesis. The results showed an increase in MVD in OSF cases compared to normal but it was statistically insignificant. Nevertheless the study did not show significantly decreased vascularity of the underlying connective tissue compared to controls. Therefore these findings challenge the conventional idea that epithelial atrophy in OSF is due to the result of poorly vascularized stroma [70]. It is further suggested that the endogenous angiogenic promoters such as iNOS, b-FGF, TGF- $\beta$, PDGF, and HIF-1 $\alpha$ that are known to be expressed in OSF, play an important role in maintaining the vascularity of underlying connective tissue [71]. After compiling all the collected data it was hypothesized that the increase in vasculature is an adaptive response of the mucosa, to cope up with the hypoxia created by progressive fibrosis. And angiogenesis in turn plays an important role in tumour proliferation, once the malignant transformation takes place [70]. Another study in which MVD was assessed using anti-factor VIII related von Willebrand factor also revealed a significant increase in MVD among OSF cases than in normal oral mucosa, providing more supporting evidence for the angiogenesis [72]. In accordance with the above findings a different group reported that more or less same MVD in OSF samples, when compared to normal healthy mucosa by hematoxylin and eosin stain. They furthermore demonstrated an increase in mean vascular luminal diameter as the disease progresses which they assumed to be an adaptive response to compensate tissue hypoxia [73]. A study based on inducible nitric oxide synthase (iNOS) reports a significant vasodilatation in the OSF cases. Another assumption forwarded with the same study is that iNOS being a powerful cytotoxic and genotoxic agent, enhanced expression of iNOS in the disease probably exerts effect on epithelial keratinocytes leading to epithelial thinning [71]. With the available literature, vascularity in the corium of OSF cases is not consistent. However, it is a well known feature in conventional histology that the vascularity of the mucosa reduces with the advance fibrosis. Further, vascularity starts to increase in the juxtaepithelial area once dysplasia appears in the epithelium. That may be part of angiogenesis related to carcinogenesis.

\section{Contribution of Integrin $\alpha v \beta \quad 6$ in Malignant Transformation}

av $\beta 6$ integrin is known for its ability to promote tissue fibrosis as well as carcinoma invasion. Considering its high expression in OSF we designed a study in order to identify the functional role of $\alpha v \beta 6$ both in vitro and in vivo. In this study it was found that arecoline, the major alkaloid of arecanut, upregulates $\alpha v \beta 6$ expression in oral keratinocytes, a process which was modulated through M4 muscarinic acetylcholine receptor, for which arecoline is an agonist. The same process could be suppressed by the M4 antagonist tropicamide. However the precise regulatory mechanism of $\alpha v \beta 6$ expression is not yet fully understood. It has been claimed that cytokines TNF- $\alpha$, TGF- $\beta 1$ and high cell density have a role to play. Since TNF- $\alpha$ production has been shown to upregulate with arecoline, TNF- $\alpha$ is most probable to form part of the downstream arecoline/M4 signaling pathway. This arecoline dependent av $\beta 6$ up-regulation in oral keratinocytes was found to promote keratinocytes migration and induce invasion, highlighting the role of integrin in malignant transformation of OSF. Further emphasizing the above fact it has been reported that over $80 \%$ of OSCCs arising on a background of OSF had moderate to high $\alpha v \beta 6$ expression [26].

EMT a process that contributes to tumour cell invasion is a newly forwarded concept that has gained substantial attention recently. TGF- $\beta 1$ and Ras may modulate the EMT and also OSCCs in OSF patients have a higher incidence of Ras mutations. Hence in the future it would be interesting to find out whether $\alpha v \beta 6$ expressing OSF lesions transform on acquiring mutated Ras and then undergo EMT [26].

\section{Senescence and Epithelial Mesenchymal Interactions}

Although the senescence growth arrest is clearly tumour suppressive, there is mounting evidence that the Senescence Associated Secretory Phenotype (SASP) can promote malignant phenotypes. It has also been shown that the SASP is a potent inducer of an epithelial-tomesenchyme transition, a critical step in the development of invasive and metastatic carcinoma. Further the above activity is due mainly to the SASP component factors IL-6, IL-8 and GRO- $\alpha$ [74]. In one of our previous studies it was clearly evident that arecoline dependent IL-6, IL-8 and GRO- $\alpha$ in cell culture leading to DNA double strand breaks through ROS. We demonstrated the similar findings in OSF tissue confirming the above statement and showed the contribution of these inflammatory molecules in malignant transformation [64].

Out of many of our recent investigations on OSF, the study designed to examine the mechanism of senescence is of utmost importance. OSF biopsies at different stages of the disease progression were analyzed, using a combination of in situ immunodetection techniques, drug treatments, fluorescence-activated cell sorting and enzyme-linked absorbance assays. Senescent fibroblasts were found to be accumulated with progression of the disease. The senescence of activated fibroblasts in OSF was mediated by intrinsic generation of ROS probably as a result of dysfunctional mitochondria, and was associated with oxidative damage, DNA damage foci and p16INK4A accumulation. Interestingly it was noted that neither the continuous presence of the disease stimulus, nor any other cell type or the tissue environment has any association with the process of senescence. Hence it was convinced that the senescent fibroblasts accumulation during the progression of OSF is due to an intrinsic property of the disease-associated fibroblast population. In contrast to the epithelial cells in which ROS were thought to act directly to accelerate senescence by causing telomere shortening and then the fibrosis, the fibroblasts achieve senescence via a telomere and cell division independent mechanism. Furthermore it was observed that the senescent fibroblasts produce 25-83 times more MMP- 1 and -2 than their pre-senescent counterparts. Therefore, senescent cells may locally ameliorate the fibrosis by increased 
expression of MMPs prior to their clearance by the innate immune system [75]. Although, our two studies show slightly different findings in relation to senescence and arecoline, increase amount of senescent cells appeared to be common. Therefore we believe in the fact that in OSF senescence related epithelial-to-mesenchyme transition takes place through SASP predisposing to carcinogenesis.

We have conducted another study on cytokeratin profiles of OSF to support epithelial mesenchymal transition. Previous studies have provided convincing evidence that keratinocyte differentiation, proliferation, migration and invasion are influenced by epithelialmesenchymal interaction. And it also had been identified that the changes in the mesenchyme result in alterations in keratin expression. The prime feature of OSF is the excessive fibrosis of subepithelial connective tissue and this abnormal mesenchyme in turn could possibly affect the overlying epithelium. In this study we hypothesized that in OSF, keratinocytes in the epithelium could be altered in such a manner that the altered keratin profiles would influence the disease pathogenesis, as well as the predisposition to malignant transformation. There was increase $\mathrm{K} 1$ and $\mathrm{K} 10$ in the suprabasal layers along with an induction of K6 in the basal layer and complete loss of K19 in the epithelium. Furthermore, there was increased K17 expression in the suprabasal layers, which correlated with histological grading of disease severity. The most significant K17 expression was observed in the most advanced cases. Although K17 expression of the basal layer was completely lost in a subset of most severe cases, there was no detectable correlation between basal K17 expression and disease severity. According to the findings of this study, existence of an OSF-specific keratinocytes phenotype with a different keratin expression profile from other cutaneous and oral diseases was clearly evident. Hence we proposed that the altered keratin profiles could be useful as histological diagnostic markers in both pathogenesis and predisposition to malignancy in OSF [76].

\section{Genetic Susceptibility}

Since OSF is considered as a high-risk pre-cancerous condition, knowledge of genetic susceptibility to malignant transformation is of immense importance, especially in developing cancer predictive markers for OSF patients. The first ever attempt to detect the genomic instability in OSF was carried out by employing the high-resolution genome-wide Affymetrix Mapping single nucleotide polymorphism microarray technique by our group. Genomic instability denotes early genetic events during malignant transformation causing Loss of Heterozygosity $(\mathrm{LOH})$ and chromosomal copy number abnormality. With this study we were able to identify a small number of discrete hotspot LOH loci in $47-53 \%$ of the OSF samples studied. The degree of LOH was appeared to have a significant positive relationship with OSF grade. The fact that many of these $\mathrm{LOH}$ loci were previously identified regions of genomic instability associated with carcinogenesis of head and neck squamous cell carcinomas (HNSCC) further emphasizes the value of these findings. The results highlight 14 chromosomes containing 23 hotspot $\mathrm{LOH}$ loci, among which chromosome 13 was found to contain the largest, in proportion to chromosomal size, $\mathrm{LOH}$ regions ranging from $13 \mathrm{q} 14$ to $13 \mathrm{q} 33$. Considering the well known fact that chromosome $13 \mathrm{q}$ is highly susceptible to genomic instability in HNSCC, we hypothesized that genes within the 13q14-q33 LOH region found in the OSF may play essential roles in the initiation of oral carcinogenesis in these patients. Other $\mathrm{LOH}$ loci revealed in this study with previously identified susceptibility regions in HNSCC include 3p24-p22, 6q26-q27, 9q22.3, 12p11.2, and 20p12-11.These LOH loci were found to harbor numerous oncogenes and tumour suppressor genes involved in the regulation of hypoxia, cellular adhesion/migration, matrix remodeling, cell cycle/apoptosis, DNA repair and inflammation. This study provides first and foremost evidence that genomic instability in the form of $\mathrm{LOH}$ is present in OSF. This acquisition of LOH may subsequently alter gene function and expression. We hypothesize that increasing LOH may play a role in OSF progression and may be responsible for malignant transformation in a subset of patients with the disease [77]. Further detailed studies targeting these genes may help in the proper understanding of malignant transformation in the background of fibrosis.

\section{Conclusions}

This review focused mainly on the literature available from 2005 up to date as we have produced a comprehensive review on the subject in 2005 [1]. It appears that we have a wealth of scientifically sound evidence on various aspects of pathogenesis and malignant transformation of the deadly disease without a definite treatment. ROS is found to play a significant role in both pathogenesis and malignant transformation. Therefore, antioxidants should have significant therapeutic benefits in managing the disease and preventing carcinogenesis in the background of fibrosis. Future research should also focus on targeting various molecules and pathways which have been identified, in order to search for effective treatment as morbidity and mortality is significantly higher in OSF.

\section{References}

1. Tilakaratne WM, Klinikowski MF, Saku T, Peters TJ, Warnakulasuriya S (2006) Oral submucous fibrosis: review on aetiology and pathogenesis. Oral Oncol 42: 561-568.

2. Deng YT, Chen HM, Cheng SJ, Chiang CP, Kuo MY (2009) Arecolinestimulated connective tissue growth factor production in human buccal mucosa fibroblasts: Modulation by curcumin. Oral Oncol 45: e99-99e105.

3. Xia L, Tian-You L, Yi-Jun G, Dong-Sheng T, Wen-Hui L (2009) Arecoline and oral keratinocytes may affect the collagen metabolism of fibroblasts. $\mathrm{J}$ Ora Pathol Med 38: 422-426.

4. Li X, Ling TY, Gao YJ (2007) Effect of arecoline on the differentiation of myofibroblasts of oral mucosa. Zhonghua Kou Qiang Yi Xue Za Zhi 42: 423427

5. Chang MC, Lin LD, Wu HL, Ho YS, Hsien HC, et al. (2013) Areca nut-induced buccal mucosa fibroblast contraction and its signaling: a potential role in oral submucous fibrosis--a precancer condition. Carcinogenesis 34: 1096-1104.

6. Thangjam GS, Agarwal P, Khan I, Verma UP, Balapure AK, et al. (2009) Transglutaminase-2 regulation by arecoline in gingival fibroblasts. J Dent Res 88: $170-175$

7. Tseng SK, Chang MC, Su CY, Chi LY, Chang JZ, et al. (2012) Arecoline induced cell cycle arrest, apoptosis, and cytotoxicity to human endothelial cells. Clin Oral Investig 16: 1267-1273.

8. Rasika Pawiththra Illeperuma, Mi Heon Ryu, Ki Yeol Kim, WM Tilakaratne, Jin $\mathrm{Kim}$ (2010) Relationship of fibrosis and the expression of TGF-ß1, MMP-1, and TIMP-1 with epithelial dysplasia in oral submucous fibrosis. Oral Med Pathol 15: 25-32.

9. Pitiyage GN, Lim KP, Gemenitzidis E, Teh MT, Waseem A, et al. (2012) Increased secretion of tissue inhibitors of metalloproteinases 1 and 2 (TIMPs-1 and -2$)$ in fibroblasts are early indicators of oral sub-mucous fibrosis and ageing. J Oral Pathol Med 41: 454-462.

10. Lin HJ, Lin JC (2007) Treatment of oral submucous fibrosis by collagenase: effects on oral opening and eating function. Oral Dis 13: 407-413.

11. Mishra G, Ranganathan K (2010) Matrix metalloproteinase-1 expression in oral submucous fibrosis: an immunohistochemical study. Indian J Dent Res 21: $320-325$.

12. Khan S, Chatra L, Prashanth SK, Veena KM, Rao PK (2012) Pathogenesis of oral submucous fibrosis. J Cancer Res Ther 8: 199-203.

13. Shieh TM, Tu HF, Ku TH, Chang SS, Chang KW, et al. (2009) Association 
Citation: Ekanayaka RP, Tilakaratne WM (2013) Oral Submucous Fibrosis: Review on Mechanisms of Pathogenesis and Malignant Transformation. J Carcinogene Mutagene S5: 002. doi:10.4172/2157-2518.S5-002

between lysyl oxidase polymorphisms and oral submucous fibrosis in older male areca chewers. J Oral Pathol Med 38: 109-113.

14. Raja KB, Hazarey VK, Peters TJ, Warnakulasuriya S (2007) Effect of areca nut on salivary copper concentration in chronic chewers. Biometals 20: 43-47.

15. Ayinampudi BK, Narsimhan M (2012) Salivary copper and zinc levels in oral pre-malignant and malignant lesions. J Oral Maxillofac Pathol 16: 178-182.

16. Khanna SS, Karjodkar FR (2006) Circulating immune complexes and trace elements (Copper, Iron and Selenium) as markers in oral precancer and cancer : a randomised, controlled clinical trial. Head Face Med 2: 33.

17. Tadakamadla J, Kumar S, GP M (2011) Evaluation of serum copper and iron levels among oral submucous fibrosis patients. Med Oral Patol Oral Cir Bucal 16: e870-873.

18. Utsunomiya H, Tilakaratne WM, Oshiro K, Maruyama S, Suzuki M, et al. (2005) Extracellular matrix remodeling in oral submucous fibrosis: its stage-specific modes revealed by immunohistochemistry and in situ hybridization. J Oral Pathol Med 34: 498-507.

19. Yang SF, Tsai $\mathrm{CH}$, Chang YC (2008) The upregulation of heat shock protein 47 expression in human buccal fibroblasts stimulated with arecoline. J Oral Pathol Med 37: 206-210.

20. Chung-Hung T, Shun-Fa Y, Yu-Chao $C$ (2007) The upregulation of cystatin $C$ in oral submucous fibrosis. Oral Oncol 43: 680-685.

21. Shetty SR, Babu SG, Kumari S, Rao V, Vijay R, et al. (2012) Malondialdehyde levels in oral sub mucous fibrosis: a clinicopathological and biochemical study. N Am J Med Sci 4: 125-128.

22. Khan I, Agarwal P, Thangjam GS, Radhesh R, Rao SG, et al. (2011) Role of TGF- $\left.\right|^{2}$ and BMP7 in the pathogenesis of oral submucous fibrosis. Growth Factors 29: 119-127

23. Rajalalitha P, Vali S (2005) Molecular pathogenesis of oral submucous fibrosis-a collagen metabolic disorder. J Oral Pathol Med 34: 321-328.

24. Chang JZ, Yang WH, Deng YT, Chen HM, Kuo MY (2013) EGCG blocks TGFÎ 21 -induced CCN2 by suppressing JNK and p38 in buccal fibroblasts. Clin Oral Investig 17: 455-461.

25. Khan I, Kumar N, Pant I, Narra S, Kondaiah P (2012) Activation of TGF-î pathway by areca nut constituents: a possible cause of oral submucous fibrosis. PLoS One 7: e51806.

26. Moutasim Karwan A, Jenei Veronika, Sapienza Karen, Marsh Daniel, Weinreb Paul H., et al. (2011) Betel-derived alkaloid up-regulates keratinocyte alphavbeta6 integrin expression and promotes oral submucous fibrosis. Journal of Pathology 223: 366-377.

27. Kale AD, Mane DR, Shukla D (2013) Expression of transforming growth factor $\beta$ and its correlation with lipodystrophy in oral submucous fibrosis: an immunohistochemical study. Med Oral Patol Oral Cir Bucal 18: e12-18.

28. Bishen KA, Radhakrishnan R, Satyamoorthy K (2008) The role of basic fibroblast growth factor in oral submucous fibrosis pathogenesis. J Oral Patho Med 37: 402-411.

29. Tsai CH, Yang SF, Chen YJ, Chou MY, Chang YC (2005) The upregulation of insulin-like growth factor-1 in oral submucous fibrosis. Oral Oncol 41: 940-946.

30. Chiu CJ, Chiang CP, Chang ML, Chen HM, Hahn LJ, et al. (2001) Association between genetic polymorphism of tumor necrosis factor-alpha and risk of ora submucous fibrosis, a pre-cancerous condition of oral cancer. J Dent Res 80 2055-2059.

31. Yanjia H, Xinchun J (2007) The role of epithelial-mesenchymal transition in oral squamous cell carcinoma and oral submucous fibrosis. Clin Chim Acta 383: 51-56.

32. Mithani SK, Mydlarz WK, Grumbine FL, Smith IM, Califano JA (2007) Molecular genetics of premalignant oral lesions. Oral Dis 13: 126-133.

33. Hu Y, Jian X, Peng J, Jiang X, Li N, et al. (2008) Gene expression profiling of oral submucous fibrosis using oligonucleotide microarray. Oncol Rep 20: 287-294

34. Hu YJ, Jian XC, Liu BJ, Peng JY (2008) Application of bioinformatics tools in analysis of differentially expressed genes in oral submucosal fibrosis. Zhonghua Kou Qiang Yi Xue Za Zhi 43: 168-171.

35. Joshi MS, Verma Y, Gautam AK, Parmar G, Lakkad BC, et al. (2011)
Cytogenetic alterations in buccal mucosa cells of chewers of areca nut and tobacco. Arch Oral Biol 56: 63-67.

36. Joshi MS, Verma Y, Gautam AK, Shivgotra VK, Parmar G, et al. (2011) Assessment of genetic damage among chewers of mixture containing mainly areca nut and tobacco. Asia Pac J Public Health 23: 852-860.

37. Li N, Hu Q, Jiang C, Hu Y, Yuan Y, et al. (2011) Novel genetic biomarkers for susceptibility to oral submucous fibrosis: cytochrome P450 3A. Med Hypotheses 77: 834-836.

38. Chaudhuri SR, Mukherjee S, Paul RR, Haldar A, Chaudhuri K (2013) CYP1A and CYP2E1 gene polymorphisms may increase susceptibility to Ora Submucous Fibrosis among betel quid chewers of Eastern India. Gene 513 . 268-271.

39. Ghosh T, Gupta S, Bajpai P, Agarwal D, Agarwal M, et al. (2012) Association of CYP1A1, GSTM1, and GSTT1 gene polymorphism with risk of oral submucous fibrosis in a section of North Indian population. Mol Biol Rep 39: 9383-9389.

40. Xie H, Liu J, Ling TY (2012) Expression of cytochrome P450 related genes in oral submucous fibrosis tissue. Zhonghua Kou Qiang Yi Xue Za Zhi 47: 743747

41. Rajendran R, Harish RK, Anil S, Vidyadharan R, Banerjee M (2010) Transforming growth factor- $\beta-1$ polymorphisms are infrequent but exist at selected loci in oral submucous fibrosis. Indian J Dent Res 21: 413-419.

42. Chaudhary AK, Singh M, Bharti AC, Singh M, Shukla S, et al. (2010) Synergistic effect of stromelysin-1 (matrix metalloproteinase-3) promoter $(-11715 \mathrm{~A}->6 \mathrm{~A})$ polymorphism in oral submucous fibrosis and head and neck lesions. BMC Cancer 10: 369

43. Chaudhary AK, Pandya S, Mehrotra R, Singh M, Singh M (2011) Role of functional polymorphism of matrix metalloproteinase-2 (-1306 C/T and -168 $\mathrm{G} / \mathrm{T}$ ) and MMP-9 (-1562 C/T) promoter in oral submucous fibrosis and head and neck squamous cell carcinoma in an Indian population. Biomarkers 16 : 577-586.

44. Mukherjee S, Bhowmik AD, Roychoudhury P, Mukhopadhyay K, Ray JG, et al. (2012) Association of XRCC1, XRCC3, and NAT2 polymorphisms with the risk of oral submucous fibrosis among eastern Indian population. J Oral Pathol Med 41: 292-302.

45. Thangjam GS, Kondaiah P (2009) Regulation of oxidative-stress responsive genes by arecoline in human keratinocytes. J Periodontal Res 44: 673-682.

46. Lee PH, Chang MC, Chang WH, Wang TM, Wang YJ, et al. (2006) Prolonged exposure to arecoline arrested human KB epithelial cell growth: regulatory mechanisms of cell cycle and apoptosis. Toxicology 220: 81-89.

47. Hsue SS, Wang WC, Chen CH, Lin CC, Chen YK, et al. (2007) Malignant transformation in 1458 patients with potentially malignant oral mucosa disorders: a follow-up study based in a Taiwanese hospital. J Oral Pathol Med 36: $25-29$

48. Chaturvedi P, Vaishampayan SS, Nair S, Nair D, Agarwal JP, et al. (2012) Ora squamous cell carcinoma arising in background of oral submucous fibrosis: A clinicopathologically distinct disease. Head Neck .

49. Guo F, Jian XC, Zhou SH, Li N, Hu YJ, et al. (2011) A retrospective study of ora squamous cell carcinomas originated from oral submucous fibrosis. Zhonghua Kou Qiang Yi Xue Za Zhi 46: 494-497.

50. Lian leB, Tseng YT, Su CC, Tsai KY (2013) Progression of precancerous lesions to oral cancer: results based on the Taiwan National Health Insurance Database. Oral Oncol 49: 427-430.

51. Muttagi SS, Chaturvedi P, Gaikwad R, Singh B, Pawar P (2012) Head and neck squamous cell carcinoma in chronic areca nut chewing Indian women: Case series and review of literature. Indian J Med Paediatr Oncol 33: 32-35.

52. Lin KH, Lin CY, Liu CC, Chou MY, Lin JK (2011) Arecoline N-oxide: its mutagenicity and possible role as ultimate carcinogen in areca ora carcinogenesis. J Agric Food Chem 59: 3420-3428.

53. Ji WT, Yang SR, Chen JY, Cheng YP, Lee YR, et al. (2012) Arecoline downregulates levels of p21 and p27 through the reactive oxygen species/ mTOR complex 1 pathway and may contribute to oral squamous cell carcinoma. Cancer Sci 103: 1221-1229.

54. Choudhury Y, Sharan RN (2011) Altered BRCA1 and BRCA2 responses and mutation of BRCA1 gene in mice exposed chronically and transgenerationally to aqueous extract of betel nut (AEBN). Environ Toxicol Pharmacol 31: 57-69. 
Citation: Ekanayaka RP, Tilakaratne WM (2013) Oral Submucous Fibrosis: Review on Mechanisms of Pathogenesis and Malignant Transformation. J Carcinogene Mutagene S5: 002. doi:10.4172/2157-2518.S5-002

55. Gao YJ, Ling TY, Yin XM, Li X, Huang Y (2007) [Effects of arecoline and nicotine on the expression of hTERT in oral keratinocytes]. Zhonghua Kou Qiang Yi Xue Za Zhi 42: 26-30.

56. Pandey M, Prakash O, Santhi WS, Soumithran CS, Pillai RM (2008) Overexpression of COX-2 gene in oral cancer is independent of stage of disease and degree of differentiation. Int J Oral Maxillofac Surg 37: 379-383.

57. Hu CW, Chao MR (2012) Direct-acting DNA alkylating agents present in aqueous extracts of areca nut and its products. Chem Res Toxicol 25: 23862392.

58. Jeng JH, Chang MC, Hahn LJ (2001) Role of areca nut in betel quid-associated chemical carcinogenesis: current awareness and future perspectives. Oral Oncol 37: 477-492

59. Rasika P Illeperuma, Young Jin Park, Jinmi Kim, Da-Woon Jung, WM Tilakaratne, et al. (2010) Cytokine expression by the treatment of areca nut extract in cultured fibroblasts: comparision between normal oral fibroblasts and hTERT transfected fibroblasts. 15th International Congress on Oral Pathology and Medicine, Seoul, Korea.

60. Tilakaratne WM, Iqbal Z, Teh MT, Ariyawardana A, Pitiyage G, et al. (2008) Upregulation of HIF-1alpha in malignant transformation of oral submucous fibrosis. J Oral Pathol Med 37: 372-377.

61. Jayasooriya PR, Nadeeka Jayasinghe KA and Mudiyanselage Tilakaratne W (2011) Relationship between thickness of fibrosis and epithelial dysplasia in oral submucous fibrosis. Journal of Investigative and Clinical Dentistry 2: 171-175.

62. Zhou SH, Li LL, Jian XC, Wang Y, Chen XQ, et al. (2008) [Molecules of $\mathrm{G}(2) / \mathrm{M}$ phase and the phosphorylation of survivin in the carcinogenesis of oral submucosal fibrosis]. Zhonghua Kou Qiang Yi Xue Za Zhi 43: 709-712.

63. Zhou S, Li L, Jian X, Ou X, Jiang H, et al. (2008) The phosphorylation of survivin Thr34 by p34cdc2 in carcinogenesis of oral submucous fibrosis. Onco Rep 20: 1085-1091.

64. Zhou S, Qu X, Yu Z, Zhong L, Ruan M, et al. (2010) Survivin as a potential early marker in the carcinogenesis of oral submucous fibrosis. Oral Surg Oral Med Oral Pathol Oral Radiol Endod 109: 575-581.

65. Angadi PV, Krishnapillai R (2012) Evaluation of PTEN immunoexpression in oral submucous fibrosis: role in pathogenesis and malignant transformation. Head Neck Pathol 6: 314-321.

66. Yin X, Wen C, Han Y, Gao Y, Tang Z (2010) Expression of FHIT and MDM2 in oral submucous fibrosis and canceration tissues. Zhong Nan Da Xue Xue Bao Yi Xue Ban 35: 572-575.

67. Li N, Jian XC, Xu CJ (2009) Expression of loricrin and cytochrome P450 3A5 in oral submucous fibrosis and their significance. Hua Xi Kou Qiang Yi Xue Za Zhi 27: 29-33.

68. Xu C, Zhao J, Loo WT, Hao L, Wang M, et al. (2012) Correlation of epigenetic change and identification of risk factors for oral submucous fibrosis. Int $\mathrm{J}$ Biol Markers 27: e314-321.

69. Lin YC, Huang HI, Wang LH, Tsai CC, Lung O, et al. (2008) Polymorphisms of COX-2 $-765 \mathrm{G}>\mathrm{C}$ and $\mathrm{p} 53$ codon 72 and risks of oral squamous cell carcinoma in a Taiwan population. Oral Oncol 44: 798-804

70. Desai RS, Mamatha GS, Khatri MJ, Shetty SJ (2010) Immunohistochemical expression of CD34 for characterization and quantification of mucosal vasculature and its probable role in malignant transformation of atrophic epithelium in oral submucous fibrosis. Oral Oncol 46: 553-558.

71. Rajendran R, Varkey S (2007) Inducible nitric oxide synthase expression is upregulated in oral submucous fibrosis. Indian J Dent Res 18: 94-100.

72. Sabarinath B, Sriram G, Saraswathi TR, Sivapathasundharam B (2011) Immunohistochemical evaluation of mast cells and vascular endothelia proliferation in oral submucous fibrosis. Indian J Dent Res 22: 116-121.

73. Rajendran R, Paul S, Mathews PP, Raghul J, Mohanty M (2005) Characterisation and quantification of mucosal vasculature in oral submucous fibrosis. Indian $\mathrm{J}$ Dent Res 16: 83-91.

74. Campisi J, Andersen JK, Kapahi P, Melov S (2011) Cellular senescence: a link between cancer and age-related degenerative disease? Semin Cancer Biol 21 354-359.

75. Pitiyage GN, Slijepcevic P, Gabrani A, Chianea YG, Lim KP, et al (2011) Senescent mesenchymal cells accumulate in human fibrosis by a telomere-independent mechanism and ameliorate fibrosis through matrix metalloproteinases. J Pathol 223: 604-617.

76. Lalli A, Tilakaratne WM, Ariyawardana A, Fitchett C, Leigh IM, et al. (2008) An altered keratinocyte phenotype in oral submucous fibrosis: correlation of keratin K17 expression with disease severity. J Oral Pathol Med 37: 211-220.

77. Teh MT, Tilakaratne WM, Chaplin T, Young BD, Ariyawardana A, et al. (2008) Fingerprinting genomic instability in oral submucous fibrosis. J Oral Pathol Med 37: $430-436$
This article was originally published in a special issue, Oncogenomics of the Head and Neck handled by Editor(s). Dr. Rui Amaral Mendes, Portuguese Catholic University, Portugal 